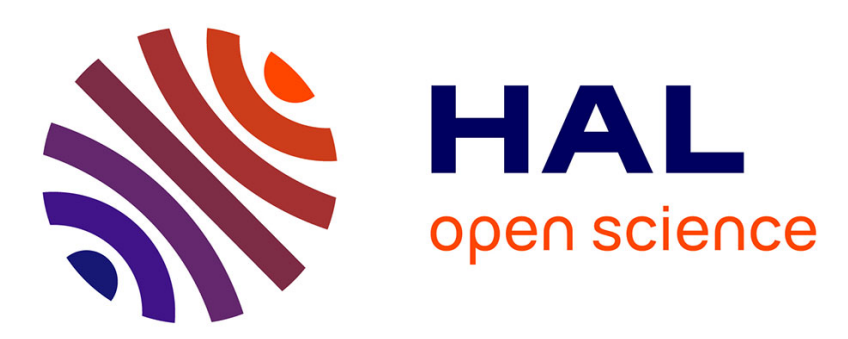

\title{
Les temples hindous et le développement local en Inde du Sud : L'exemple du Tamil Nadu
}

\author{
Pierre-Yves Trouillet
}

\section{To cite this version:}

Pierre-Yves Trouillet. Les temples hindous et le développement local en Inde du Sud: L'exemple du Tamil Nadu. L'Information géographique, 2016, religion, 80, pp.76-104. 10.3917/lig.801.0076 . hal-01535648

\author{
HAL Id: hal-01535648 \\ https://hal.science/hal-01535648
}

Submitted on 21 Oct 2020

HAL is a multi-disciplinary open access archive for the deposit and dissemination of scientific research documents, whether they are published or not. The documents may come from teaching and research institutions in France or abroad, or from public or private research centers.
L'archive ouverte pluridisciplinaire HAL, est destinée au dépôt et à la diffusion de documents scientifiques de niveau recherche, publiés ou non, émanant des établissements d'enseignement et de recherche français ou étrangers, des laboratoires publics ou privés. 


\title{
LES TEMPLES HINDOUS ET LE DÉVELOPPEMENT LOCAL EN INDE DU SUD. L'EXEMPLE DU TAMIL NADU
}

\author{
Pierre-Yves Trouillet
}

Armand Colin | « L'Information géographique »

2016/1 Vol. 80 | pages 76 à 104

ISSN 0020-0093

ISBN 9782200930516

Article disponible en ligne à l'adresse :

https://www.cairn.info/revue-l-information-geographique-2016-1-page-76.htm

Distribution électronique Cairn.info pour Armand Colin.

(C) Armand Colin. Tous droits réservés pour tous pays.

La reproduction ou représentation de cet article, notamment par photocopie, n'est autorisée que dans les limites des conditions générales d'utilisation du site ou, le cas échéant, des conditions générales de la licence souscrite par votre établissement. Toute autre reproduction ou représentation, en tout ou partie, sous quelque forme et de quelque manière que ce soit, est interdite sauf accord préalable et écrit de l'éditeur, en dehors des cas prévus par la législation en vigueur en France. Il est précisé que son stockage dans une base de données est également interdit. 


\title{
Les temples hindous et le développement local en Inde du Sud. L'exemple du Tamil Nadu
}

\author{
Par Pierre-Yves Trouillet
}

Pierre-Yves Trouillet, Géographe, chargé de recherche au CNRS, Centre d'Études de l'Inde et de l'Asie du Sud (UMR 8564 EHESS/CNRS) - pytrouillet@gmail.com

Depuis plus de quinze siècles, les temples ( $k \overline{o y} y i l$ ou kōvil en tamoul) sont des lieux d'une importance majeure pour la société tamoule en Inde du $\mathrm{Sud}^{1}{ }^{2}$ Il s'agit certes de lieux où l'on prie les dieux, mais ce sont aussi souvent de véritables pôles sociaux, économiques et politiques où s'affichent les différences de statut, que ce soit lors des rituels ou par le biais de dons ostensiblement offerts à la divinité tutélaire du lieu saint.

On trouve aujourd'hui des temples hindous dans toutes les villes et villages du Tamil Nadu (le «pays tamoul », figure 1), allant des petits autels et sanctuaires de rue aux grands centres de pèlerinage ${ }^{3}$. Beaucoup sont même devenus de riches établissements grâce aux dons monétaires et fonciers qu'ils reçoivent depuis des siècles.

On observe également depuis une trentaine d'années une multiplication spectaculaire des constructions et des rénovations de temples dans le paysage du pays tamoul. Des lieux de culte hindous de toutes tailles apparaissent ainsi dans les villes et les campagnes, tandis que les sites de pèlerinage attirent de plus en plus de fidèles et connaissent une croissance urbaine rapide (comme dans d'autres régions de l'Inde ${ }^{4}$ ). Les cultes des «saints» (ou gourous ${ }^{5}$, guru) poursuivent leur essor alors que les hindous de classes et de castes

\footnotetext{
1. Cet article est issu d'un travail mené dans le cadre du programme de recherche SUBURBIN (Subaltern Urbanisation in India) financé par l'Agence Nationale de la Recherche, et coordonné par Éric Denis et Marie-Hélène Zérah.

2. L'auteur adresse ses remerciements à Éric Denis, Marie-Hélène Zérah, Amita Baviskar, Gilles Tarabout et aux évaluateurs de la revue pour leurs commentaires sur les versions préalables de ce texte.

3. Dans 1'hindouisme contemporain, les pratiques rituelles s'effectuent aussi très souvent dans les maisons, où un espace est généralement réservé au culte $(p \bar{u} j \bar{a})$ régulièrement adressé à diverses divinités, représentées par des images pieuses ou des statuettes rituelles.

4. Voir notamment Das \& Ray (2008) et Shinde \& Pinkney (2013).

5. Maîtres spirituels pouvant être reconnus comme des incarnations de divinités.
} 


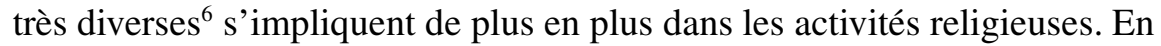
parallèle, la législation indienne permet aux institutions religieuses telles que les temples hindous, de mener de nombreuses activités caritatives et autres projets de développement local sur leurs propriétés foncières.

Ce contexte général invite à s'interroger sur le rôle que peuvent jouer les temples hindous dans le développement ${ }^{7}$ et la croissance des localités au Tamil Nadu. Cela d'autant plus que cette question semble curieusement avoir été négligée par les études urbaines indiennes en dépit de quelques travaux notables (Reiniche 1985 ; Guilmoto et al. 1989 ; Gaucher 2007 ; Viguier 2007 ; Louiset 2011 ; Shinde \& Pinkney 2013 ; Narayanan 2015). En effet, alors qu'une littérature abondante traite des dynamiques urbaines en Inde et qu'un corpus encore plus fourni porte sur les temples, les pèlerinages et autres gourous hindous, très peu d'études ont questionné les relations entre les temples et le développement local en Inde, en particulier pour la période actuelle. Les historiens (notamment Stein 1960 \& 1961 ; Heitzman 1987) s'accordent pourtant sur le rôle important joué par les temples dans l'économie agricole du pays tamoul médiéval ainsi que dans le développement de l'irrigation et de l'urbanisme. Pourquoi donc ne plus s'y intéresser pour la période contemporaine, alors que les temples se multiplient et s'enrichissent, et que les villes, tout comme l'Inde entière, connaissent une dynamique générale de croissance et de développement?

En se basant sur l'étude de plusieurs temples anciens et récents, cet article entend combler en partie ce manque en montrant comment ces institutions religieuses sont capables d'influencer et de stimuler la croissance de leur localité, et dans quelle mesure elles produisent des formes d'urbanisation in situ. Denis et al. (2012 : 56) ont récemment montré que l'urbanisation indienne se caractérise dans certains cas par une "autonomous growth of settlements generated by market and historical forces which are not "dependent" on large metropolises [...] or "planned'cities" ». Dans le prolongement de ce constat, notre intention est de montrer que les temples participent de ce type d'urbanisation (qualifiée de « subalterne ${ }^{8} »$ par les auteurs [ibid.]), d'une part en identifiant différentes situations où un temple hindou produit de la

\footnotetext{
6. Au sujet des classes moyennes urbaines, voir notamment Shinde \& Pinkney (2013) et Punzo-Waghorne (2004).

7. Le terme « développement » n'est pris ici que sous son acception la plus basique qui réfère davantage à la croissance spatiale, économique et démographique des localités qu'au développement humain ou durable, bien que beaucoup de fondations de temples hindous gèrent des activités caritatives (hôpitaux, établissements d'enseignement, etc.).

8. Les auteurs considèrent que les bourgs et petites villes constituent une strate négligée du système de villes comparativement aux grandes agglomérations et aux processus de métropolisation, qui ne sauraient pourtant résumer à eux seuls les phénomènes d'urbanisation, en Inde notamment.
} 
centralité et accompagne le développement local, et d'autre part en exposant la diversité des relations possibles entre les temples et la croissance des localités au Tamil Nadu. De fait, un autre objectif est de rappeler le rôle de l'« interdit spatial de propriété » attribué aux lieux sacrés dans l'émergence et la structuration des localités (Desmarais 2001). En effet, la géographie structurale considère que les établissements humains naissent universellement à partir de la fondation de lieux sacrés, espaces non aliénables autour desquels s'opère la transformation de l'environnement naturel en espaces culturellement investis (ibid.). Enfin, l'article illustre également l'intérêt du courant émergent de l' «économie religieuse » (Iannacone 1998; Obadia 2013) pour comprendre le rôle joué par les temples dans les territoires : ces institutions ne sont pas simplement des lieux symboliques, mais aussi, parfois, de véritables acteurs des économies locales.

Dans cette perspective, un travail de terrain a été conduit en 2012 et 2013 dans deux types de localités (Figure 1). Un premier ensemble de trois villes de moins de 100000 habitants, dans lesquelles nous nous sommes intéressés à des temples relativement anciens ${ }^{9}$ et réputés, et aujourd'hui entièrement gérés par l'État du Tamil Nadu. La première partie de l'article porte sur ce type de temples afin de montrer dans quelle mesure ils ont pu participer - et participent encore - à la croissance et à l'économie urbaines dans le temps long. L'autre ensemble de localités étudiées concerne trois bourgs de tailles beaucoup plus modestes (moins de 5000 habitants) où des temples ont été construits récemment (entre 1977 et 2007) sur des initiatives privées. La seconde section traite de ces temples récents dans l'optique d'identifier des processus d'émergence de développement local liés à différentes stratégies d'acteurs, tels que le développement commercial et immobilier autour de temples, ou encore l'achat de terrains ou la création d'établissements médicaux et d'enseignement par les fondations gestionnaires des lieux de culte.

\section{- Les temples urbains anciens}

L'influence des temples sur le développement et la croissance des villes du Tamil Nadu a été étudiée à Tindivanam (district de Viluppuram), Tiruchengodu (district de Namakkal) et Tharamangalam (district de Salem). Tindivanam est une ville moyenne (municipality) de 71257 habitants en

9. Dans la mesure où ces temples ont été construits avant ou pendant la période coloniale (i. e. avant 1947). 
Fig. 1 : Localisation des terrains d'étude au Tamil Nadu et leur population en 2011

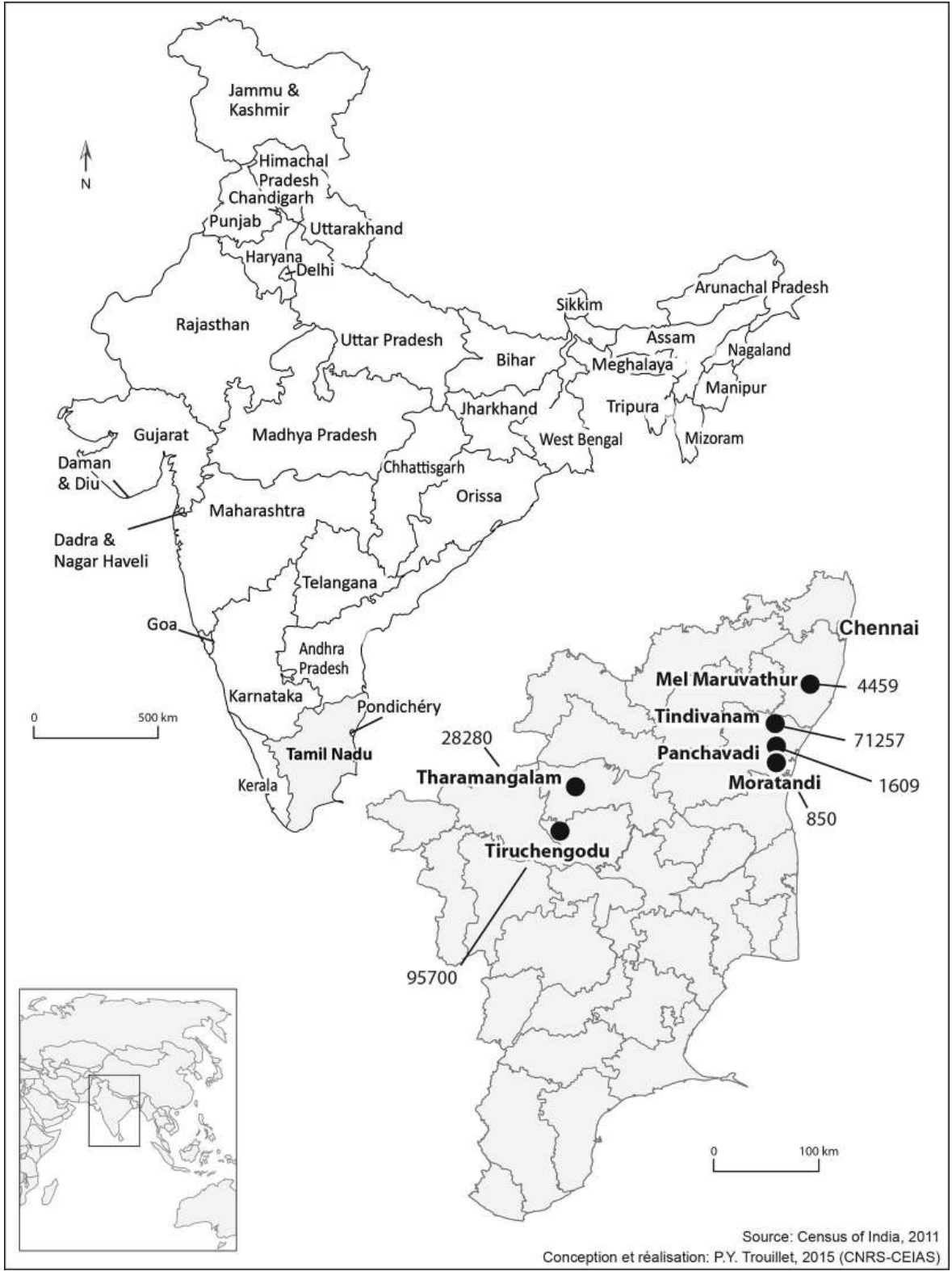


$2011^{10}$. Tiruchengodu est une autre municipality, dont la population atteignait 95700 habitants en 2011. Enfin, la ville de Tharamangalam est de dimension plus modeste, avec ses 28280 habitants recensés en 2011 et son statut de panchayat town.

Bien que cela n'ait pas été volontaire dans le choix des terrains d'étude, il s'avère que les temples anciens jouant un rôle dans la croissance et l'économie de ces trois villes sont tous dédiés au dieu Śiva (Shiva). Pour autant, cela n'est pas très surprenant - voire au contraire représentatif de la situation régionale -, puisque Śiva est le grand dieu pan-hindou le plus populaire du pays tamoul, et que son statut de dieu majeur de la « Grande ${ }^{11}$ » tradition hindoue lui vaut d'avoir des brahmanes ${ }^{12}$ pour officiants et de nombreux temples importants dans la région, car généreusement financés par les rois et les notables depuis des siècles.

Par ailleurs, tous ces temples sont gérés par l'État du Tamil Nadu, ce qui, comme nous le verrons, a son importance dans les modalités concrètes de l'implication de ces institutions religieuses dans les économies locales. En conséquence, cette partie traite en premier lieu de l'administration et du statut des temples au Tamil Nadu. La place qui revient aux temples dans les mythes fondateurs des villes est ensuite évoquée, ainsi que leur influence sur la morphologie urbaine et les activités économiques implantées sur leurs propriétés foncières.

\section{Administration et statuts des temples}

Les Britanniques s'étaient fortement impliqués dans le contrôle et l'administation des temples du sud de l'Inde, ainsi que dans la régulation de leurs ressources économiques et foncières qui pouvaient (et peuvent encore) atteindre des sommes phénoménales (Presler 1983 et 1987 ; Fuller 2003 ; Tarabout 2006). En plus de leur souci de contrôler des lieux hautement symboliques où les statuts sociaux sont régulièrement mis en scène lors de cérémonies rituelles, l'objectif était d'éviter les problèmes de gaspillage, de mauvaise gestion, de corruption et d'appropriation des «biens publics » que représentent les ressources des temples. C'est ainsi, à partir du XIX ${ }^{\mathrm{e}}$ siècle et

\footnotetext{
10. Source : Census of India.

11. Tradition de l'hindouisme fondée sur les textes et les rites brahmaniques. Elle reconnait surtout quelques grandes divinités pan-hindoues, telles que Śiva ou Viṣnu (Vishnou), dont les desservants doivent obligatoirement être des brahmanes (cf. note suivante). Paralièlement à cette « Grande » tradition orthodoxe et savante, l'hindouisme « populaire » reconnaît quant à lui toute une multitude de divinités locales, allant des déesses tutélaires de village aux divinités maléfiques, et dont les sanctuaires n'ont à l'inverse pratiquement jamais de brahmanes pour officiants.
}

12. Caste (varna) des prêtres et des érudits, la plus « haute » dans la hiérarchie sociale et rituelle hindoue. 
conjointement au déclin de la royauté en pays tamoul, que l'administration coloniale de la Présidence de Madras s'est progressivement instituée comme nouveau protecteur et administrateur des principaux temples de la région, qui sont depuis légalement reconnus comme des fondations religieuses publiques (public religious trusts) ${ }^{13}$. L'intérêt était également fiscal, puisque l'État put dès lors non seulement contrôler les ressources des temples, mais aussi prélever dans ces ressources afin de pourvoir aux dépenses nécessaires à l'entretien et au fonctionnement des lieux de culte.

Dans la continuité de cette politique introduite par les Britanniques, l'État du Tamil Nadu administre aujourd'hui plus de 36000 temples, dont ceux qui ont été étudiés à Tindivanam, Tharamangalam et Tiruchengodu. Tous ces lieux de culte sont ouverts au public, officiellement ${ }^{14}$ sans distinctions de caste ou d'appartenance sectaire, conformément à la Constitution indienne. Leurs gestionnaires (trustees et executive officers) sont généralement désignés par le ministère régional en charge de superviser ces institutions (l'Hindu Religious and Charitable Endowment Department, ou HR \& $\mathrm{CE}^{15}$ ), sauf dans le cas particulier des hereditary trustees, descendants d'anciens fondateurs de temples privés devenus publics, qui peuvent faire valoir ce droit pour participer au conseil d'administration du temple (board of trustees). Parallèlement à ce conseil, constitué, pour les temples publics, de trois à cinq membres ayant tous une autre activité professionnelle ${ }^{16}$, les executives officers sont des fonctionnaires dont le métier consiste précisément à gérer les affaires d'un temple (parfois plusieurs), à gérer son buget et son personnel (dont les prêtres) et à organiser les fêtes religieuses. Ce mode d'administration concerne la plupart des temples en activité, ouverts au public et/ou récoltant plus de 10000 roupies $^{17}$ (environ 140 euros) de dons par $a^{18}{ }^{18}$, ce qui inclut

13. Pour plus de détails sur le droit et l'administration des temples en Inde, voir notamment Varadachari (1968), Annoussamy (2001), Mukherjea (2003) et Clémentin-Ojha (2010).

14. Il arrive cependant que des discriminations persistent à l'égard des plus « basses » castes, en particulier dans les temples de rayonnement local patronnés par des castes dominantes.

15. Pour un rapport complet sur les changements dans l'administration des temples au pays tamoul entre le XIX ${ }^{\mathrm{e}}$ siècle et les années 1980, voir Presler (1983 et 1987).

16. Selon la loi, les conseils de trustees des temples publics du Tamil Nadu doivent comporter obligatoirement au moins une femme et une personne appartenant à une caste « répertoriée » (Scheduled Caste, correspondant aux « ex-Intouchables ») ou «tribale» (Scheduled Tribe). Ces conseils de trustees sont dissouts après chaque changement de gouvernement régional, pour qu'il en soit désigné de nouveaux avec des membres politiquement favorables au nouveau gouvernement.

17. Actuellement un euro équivaut à environ 70 roupies indiennes.

18. Dès qu'un temple est ouvert au public et/ou reçoit plus de 10000 roupies de dons par an, il doit être supervisé par l'HR \& CE, dont les inspecteurs vérifient les comptes chaque année. Tant qu'aucune mauvaise gestion, conflit interne ou fraude fiscale ne sont constatés, le conseil d'administration (board of trustees) intial est maintenu. Dans le cas contraire, l'HR \& CE prend le contrôle du temple et désigne un nouveau conseil ainsi qu'un executive officer (entretien avec l'assistant commissioner du bureau de l'HR \& CE de Chennai, avril 2013). 
bien sûr les établissements attirant le plus grand nombre de fidèles, les plus riches et les plus importants.

Parallèlement aux temples publics, destinés à l'usage et au bénéfice du « public » au sens large, la loi indienne reconnaît également des temples (ou fondations religieuses) privés réservés au culte d'une divinité de famille, de caste ou de communauté, et gérés par des individus privés (cas néanmoins très rare pour les grands temples). Cette distinction est importante notamment parce que les fondations de temples publics sont exonérées d'impôt de la même manière que les fondations caritatives ${ }^{19}$, alors que les fondations privées ne le sont pas. Or cette exonération permet certaines stratégies d'investissement et de développement local, puisque toute fondation de temple peut acquérir des biens fonciers, mobiliers et immobiliers. Si l'exonération est garantie pour les temples publics, les gestionnaires d'un temple privé doivent en revanche créer une fondation caritative associée au temple pour être exonérée. Ces différentes stratégies se retrouvent surtout pour les nouveaux temples qui seront évoqués dans la seconde partie.

\section{Le temple et l'origine mythique de la ville}

Un premier lien fondamental entre les temples anciens et leurs localités concerne le domaine mythe. Les travaux de l'ethnologue Marie-Louise Reiniche ont d'ailleurs montré qu'en pays tamoul, c'est « la manifestation du divin [qui] détermine la fondation d'une localité » (Reiniche 1985 : 76). En d'autres termes, aucune ville sud-indienne n'aurait été fondée au hasard (au moins dans les discours et représentations a posteriori) mais toujours à l'endroit où un mythe dit que le divin s'est manifesté, généralement sous la forme d'une statue enterrée (d'un lingam ${ }^{20}$ ou d'une déesse locale ${ }^{21}$ ) puis « découverte » un beau jour par un fidèle. L'érection d'un temple et le culte qui y est rendu sont donc pensés comme précédant l'installation humaine, par principe comme dans l'histoire locale, en correspondance directe avec les postulats de la géographie structurale évoqués en introduction.

Pour cette raison, les mythes fondateurs des villes sud-indiennes sont très souvent associés à leur temple principal. Le temple majeur de Tharamangalam en fournit un bon exemple car il aurait à sa manière « fait » la ville. Selon l'histoire du temple (sthala purāna), le site était recouvert par la

\footnotetext{
19. Néanmoins, pour pouvoir prétendre aux exonérations fiscales, les fondations religieuses publiques et les fondations caritatives ne doivent pas faire de bénéfices significatifs. Elles ne peuvent ainsi épargner plus de $25 \%$ de leurs revenus annuels et sont même censées en dépenser $85 \%$ pour réaliser les objectifs de la fondation définis lors de sa création.

20. Litt. « signe ». Pierre érigée de forme phallique signifiant la présence du dieu Śiva manifesté.

21. Cf. note 11 .
} 
jungle jusqu'à ce qu'un roi de la région y trouve un lingam et décide de fonder un temple autour de la pierre divine. Toujours selon la légende locale, il aurait fallu trois générations de rois pour achever la construction du temple autour duquel la ville s'est développée. Aujourd'hui les fidèles qui se rendent au temple peuvent expérimenter ce mythe local grâce aux trois statues de Vināyaka (Ganeśa ${ }^{22}$ qui auraient été installées par chacun des rois à l'entrée du sanctuaire.

Le fait que le temple soit à l'origine de la ville est également symbolisé dans le toponyme, puisque Tharamangalam est aussi considéré comme l'un des lieux ${ }^{23}$ (dharā) où le dieu Śiva se serait marié avec Pārvatī, la forme de l'épouse (manigalā) de la Déesse hindoue. Ce phénomène est très courant en Inde du Sud et se retrouve notamment à Tindivanam, qui doit également son toponyme à son principal temple de Śiva, manifesté localement sous le nom de «Tinti vañam īçuvaran » (le Seigneur de la forêt de tamarins).

Beaucoup d'autres exemples attestent de l'importance des temples et de leurs mythes locaux dans l'identité des villes du Tamil Nadu. C'est par exemple le cas de la célèbre Tiruvannamalai, situé à 200 kilomètres au sud de Chennai, et qui aurait été créée autour d'un lingam de feu representé par le grand inselberg dominant la ville.

\section{L'achétype de la « ville-temple » sud-indienne}

L'importance des mythes fondateurs pour l'identité et le développement des localités sud-indiennes est particulièrement marquante dans le cas des « villes-temples » (Geddes 1919). En effet, dans ce type de villes que l'on retrouve à Tiruchengodu et Tharamangalam, comme dans bien d'autres villes saintes du Tamil Nadu (Gaucher 2007 ; Viguier 2007), telles que Madurai et Tiruvannamalai, le sanctuaire principal est pensé comme le « centre protohistorique » (Guilmoto et al. 1990 : 15) de la localité, et apparaît même très nettement comme l'élément central de la structuration de la morphologie urbaine (figure 2$)^{24}$.

Comme Guilmoto, Reiniche et Pichard (ibid.) l'ont bien montré, le temple majeur des villes-temples est «le principe directeur de la morphologie urbaine ». Il «a dicté sa trame aux réseaux circulatoires et à l'habitat du

\footnotetext{
22. Célèbre dieu hindou à tête d'éléphant.
}

23. De la même manière que tout cours d'eau indien peut être considéré comme une réplique locale du grand fleuve sacré qu'est le Gange (Eck 2012), Tharamangalam est l'une des nombreuses localités dont la mythologie locale en fait un lieu du mariage de Śiva et Pārvatī.

24. Ce n'est en revanche pas le cas à Tindivanam, qui ne peut être considérée comme une ville-temple puisqu'aucun lieu de culte spécifique n'a été suffisamment important pour influencer la morphologie de la ville, mais où différents temples ont produit plusieurs centralités religieuses de moindre influence. 
Fig. 2 : La position centrale du temple de Tiruchengodu et son influence sur la morphologie urbaine

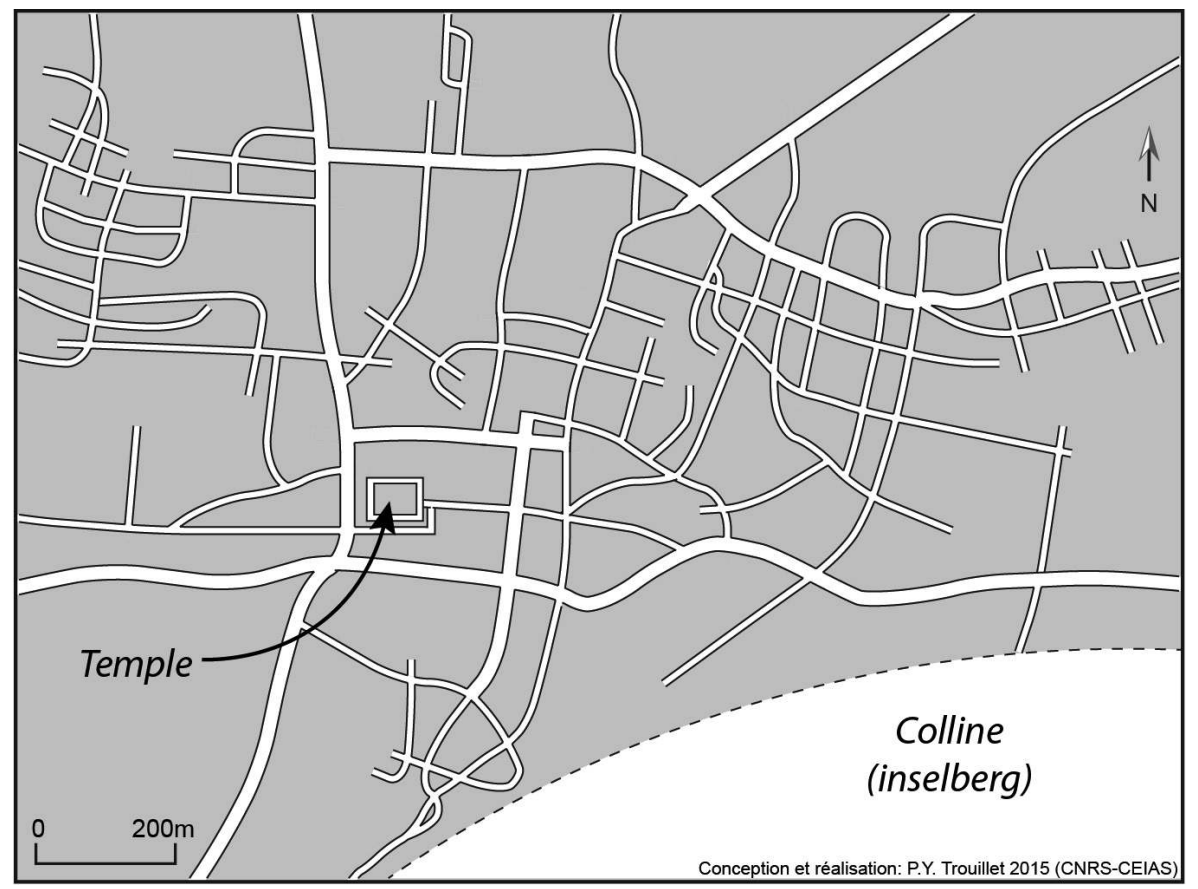

passé en structurant l'espace urbain jusqu'à la période la plus récente », il « est le point d'appui de la morphologie de la ville » et il figure comme le «point de convergence des voies de communication » (ibid. : 15-25). Néanmoins, à l'instar de ces auteurs, nous ne retenons ici que la dimension morphologique du concept de «ville-temple » car les temples structurent rarement toute l'orientation socio-économique d'une agglomération.

Ce type de villes se caractérise donc par un grand temple situé au principal croisement de la localité dont il définit le centre morphologique et surtout symbolique. Le temple détermine notamment le meilleur endroit pour le commerce de détail en raison de sa capacité à attirer de nombreux visiteurs extérieurs. À Tharamangalam et Tiruchengodu, par exemple, la plus forte concentration de petits commerces se trouve dans les rues ceinturant le temple principal, et le marché à leur proximité immédiate.

L'effet structurant des temples que l'on trouve notamment dans ce type de villes, concerne également des restrictions spatiales fondées sur des normes socio-religieuses. En effet, la vente de produits tels que la viande, l'alcool ou le tabac est proscrite près des grands temples car leur nature (guna), considérée comme « impure » (aśaiva), est incompatible avec la sainteté du 
Fig. 3 : Étals à l'entrée du grand temple de Tiruchengodu (clichés auteur, 2012)

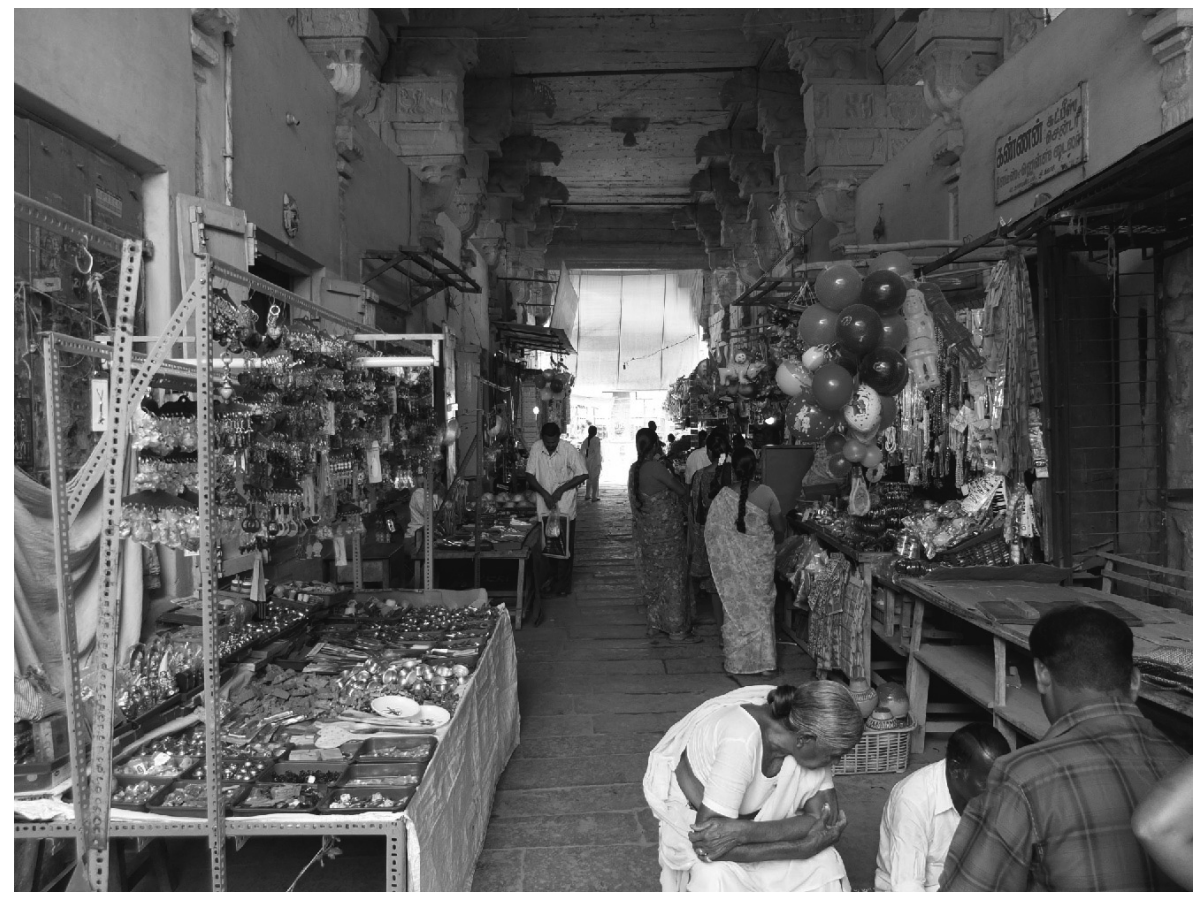

lieu de culte. Cette combinaison entre attraction et interdit autour du temple hindou se retrouve dans la structuration de l'habitat des groupes sociaux. Depuis des siècles en effet, l'habitat des castes parias (longtemps nommées « intouchables » et plus récemment « répertoriées ${ }^{25}$ » par l'administration ou dalit $^{26}$ par leurs défenseurs militants) est proscrit à proximité des grands temples alors que les plus « hautes» castes résident dans leurs environs. Dans les localités tamoules, les Brahmanes vivent ainsi habituellement à proximité du temple principal, voire dans un quartier monocaste appelé agrahāram ${ }^{27}$ lorsqu'ils sont assez nombreux. À Thiruchengodu par exemple, la rue adjacente au temple par le sud est surnommée localement Gurrukal street, ou la « rue des prêtres brahmanes ». Ces observations ne sont pas

25. L'administration indienne emploie l'expression Scheduled Castes pour désigner ces communautés défavorisées et ségréguées. L'intention est de les « répertorier » pour qu'elles puissent bénéficier de la politique de discrimination positive (quotas de postes réservés dans la fonction publique ou de places pour les étudiants dans les universités, par exemple).

26. Litt. « Opprimés ».

27. Donation royale de terres à des Brahmanes puis, finalement, quartier de résidence des Brahmanes. 
nouvelles et se retrouvent même dans des localités qui ne sont pas des « villestemples », mais elles rappellent le rôle structurant joué par les temples dans la segmentation de l'habitat et des activités commerciales, et donc dans le dessin de la morphologie des localités.

\section{Des activités sur les terres des temples}

Le rôle des temples dans le développement local est loin d'être seulement symbolique. Certaines activités économiques, comme des bâtiments commerciaux, sont directement installées sur des terrains appartenant aux temples... ou plus précisément aux divinités tutélaires des temples.

En effet le droit indien contemporain, qui s'inspire en partie du droit hindou, reconnaît les divinités comme les propriétaires de toutes les ressources - tant monétaires que foncières et immobilières - qui leur ont été offertes sous forme de dons et qui sont inaliénables ${ }^{28}$. Chaque divinité de temple est même reconnue comme ayant une personnalité juridique comparable par certains aspects à celle d'une personne mineure, dont les ressources sont gérées et administrées par des curateurs (les trustees) (Varadachari 1968 ; Presler 1987 ; Reiniche 1989 ; Annousammy 2001 ; Mukherjea 2003 ; ClémentinOjha 2010). Le temple hindou ne doit donc pas être pensé seulement comme un édifice religieux mais comme une véritable institution ou «fondation » (temple trust, ou encore devasthanam, le « lieu du dieu») associée à la divinité tutélaire du lieu de culte, ayant ses propres biens, ses gestionnaires et de nombreux employés.

Ainsi à Tharamangalam, les 44 échoppes situées dans les quatre rues ceinturant le sanctuaire principal de la ville appartiennent toutes à la fondation du temple. Ces commerces de détail vendent différents types d'articles, tels que des objets rituels, des vêtements, des produits ménagers, de la nourriture, etc. Néanmoins, aucun restaurant non-végétarien (aśaiva) n'est permis sur les terres des temples du Tamil Nadu, en raison des normes socio-religieuses qui ont été évoquées plus haut. Dans le cas de Tharamangalam, la fondation du temple possède à la fois le terrain et les bâtiments commerciaux qu'il loue par baux d'un à trois ans. Comme pour tous les autres temples publics, le montant de la location est fixé lors d'enchères. Ainsi entre 2011 et 2014, les temples publics du Tamil Nadu ont perçu plus de 2 milliards de

28. Pour plus de details sur l'histoire et la diversité des donations foncières aux temples hindous, voir notamment Colas (2009) et Tarabout (2009). 
roupies (30 millions d'euros) à partir des revenus locatifs de leurs propriétés immobilières ${ }^{29}$.

Les temples hindous possèdent également des terrains agricoles qui peuvent se situer dans leur localité ou ailleurs, loués par baux lors d'enchères dont la fréquence dépend du type de biens (bâtiments, terrain irrigué ou non, etc.). En 2014, les 36000 temples contrôlés par le HR \& CE possédaient plus de 160000 hectares de terres agricoles qui étaient loués à plus de 100000 exploitants. Ces propriétés foncières des temples peuvent être cultivées, vacantes ou encore occupées par des maisons individuelles dont les loyers sont généralement inférieurs au prix du marché. Si les temples conservent toujours le titre de propriété (pațāa), les habitations peuvent néanmoins être vendues sur un marché informel entre particuliers.

Par ailleurs, comme beaucoup de temples hindous ont un rayonnement régional voire national, il est assez fréquent que leurs propriétés foncières soient situées à plusieurs dizaines ou centaines de kilomètres de leur localité. Il s'agit alors de terrains leur ayant été offerts par des donateurs originaires d'autres régions, souvent depuis plusieurs décennies, voire plusieurs siècles pour les temples les plus anciens. C'est d'ailleurs en raison de cette atomisation des terres des temples que l'État du Tamil Nadu (comme d'autres États de l'Inde) a récemment lancé un programme destiné à collecter le maximum d'informations sur les propriétés foncières des temples publics, afin d'identifier et récupérer celles ayant été perdues ou spoliées.

L'influence des temples dans la croissance et le développement des villes tamoules est donc plus complexe qu'il n'y paraît. Il s'agit de lieux hautement symboliques contribuant non seulement à la distinction et à la définition de l'identité de leur localité, mais pouvant aussi participer à la structuration des formes urbaines grâce à leur capacité à influencer localement le dessin de la morphologie (tracé des rues) et à orienter certains usages du foncier au travers d'interdits spatiaux (habitat des castes, types de commerce). De plus, en permettant le développement d'activités économiques sur leurs propriétés foncières, les temples participent directement à l'économie locale. Voyons maintenant comment ces institutions religieuses peuvent être de véritables acteurs du développement local, à travers trois exemples de temples récemment fondés dans des bourgs et des villages.

29. Tamil Development, Religious Endowments and Information Department, Hindu Religious and Charitable Endowments Department, Demand N 47, Policy note 2014-2015, 2014, 165 p. 
Les temples hindous et le développement local en Inde du Sud

Fig. 4 : Magasins installés sur les terres des temples de Tharamangalam et de Tiruchengodu (2012)
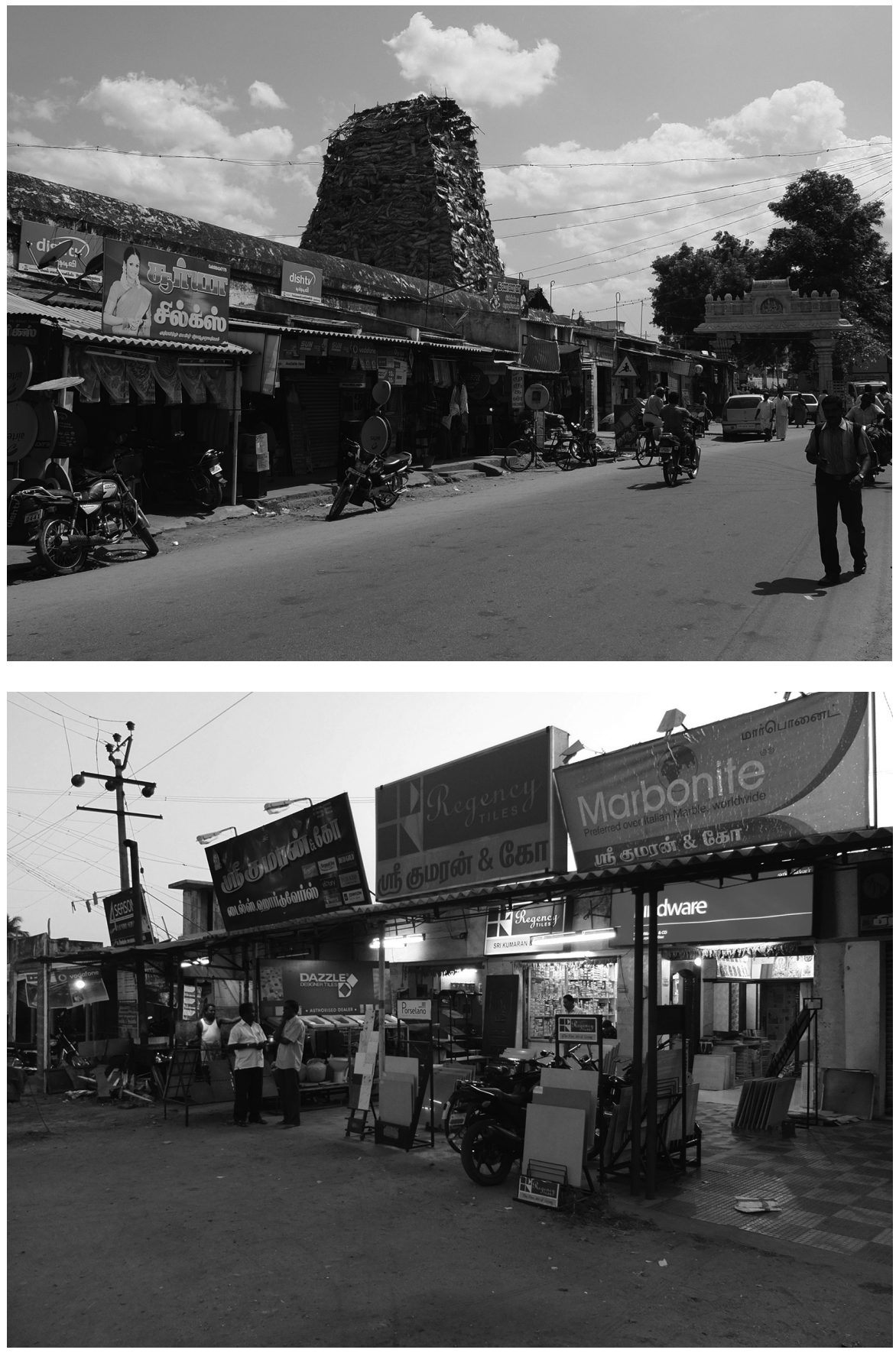


\section{- Les nouveaux temples des bourgs et des villages}

Les trois cas d'étude suivants concernent des temples beaucoup plus récents que ceux qui viennent d'être évoqués et situés dans des localités de bien moindre dimension. Ils permettent d'observer plusieurs types de motivations de fondateurs de temples contemporains, allant de l'évergétisme de notables au développement d'établissements médicaux et d'enseignement par un gourou, en passant par la stratégie économique d'un prêtre ayant créé une fondation de temple pour acquérir des terrains. Ils montrent également qu'à l'instar des temples plus anciens, les nouveaux sanctuaires hindous peuvent devenir des « hauts lieux » (landmarks) capables d'attirer des activités économiques dans leurs environs (commerce de détail, développement immobilier, etc.) et de transformer les géographies locales.

\section{Évergétisme et transformation de la géographie socio-religieuse locale}

L'évergétisme est souvent à l'origine des constructions de nouveaux temples hindous. Cette pratique, bien étudiée par Paul Veyne (1976) pour le monde gréco-romain, consiste pour les notables à conforter leur statut social en participant au bien-être de la collectivité, notamment à travers le financement de constructions de monuments, de fêtes religieuses et autres dons aux nécessiteux. En Inde, l'évergétisme exprimé dans le domaine religieux est loin d'être un phénomène nouveau, notamment dans le Sud où les rois ont eu pendant des siècles le devoir de financer et de protéger les temples, où leur statut était en retour consacré publiquement par les prêtres lors des grandes fêtes religieuses (Breckenridge \& Appadurai 1976 ; Reiniche 1985). Milton Singer (1972) a insisté sur l'importance contemporaine des pratiques religieuses pour les industriels de Madras, telles que le pèlerinage, le patronage de fêtes rituelles, ou l'aide financière aux fondations de temples et autres monastères. Clémentin-Ojha et Lachaier (2008) ont également montré que le statut social des marchands et des industriels hindous dépendait souvent non seulement de leur richesse, mais aussi de leur générosité, en particulier dans les activités religieuses. Il faut enfin souligner l'instrumentalisation de plus en plus courante des financements de constructions et de rénovations de temples par les politiciens depuis le milieu des années 1990 au Tamil Nadu (Fuller 2003) comme ailleurs, qui explique aussi en grande partie la multiplication des nouveaux temples.

Le temple de Paňcamukha Āñjanēya Svāmi (une forme de Hanumān, le dieu à tête de singe), dont l'inauguration fut célébrée en grande pompe le 31 janvier 2007 dans le petit village de Panchavadi, fournit un bon exemple de l'implication contemporaine des notables dans la création de hauts-lieux 
religieux capables de stimuler le développement de leur localité. Peuplé d'à peine 1600 âmes en 2011, ce village est situé dans les environs de Pondichéry, à proximité d'un échangeur autoroutier reliant l'ancien comptoir français à Chennai, ce qui lui confère une bonne accessibilité. Le nouveau lieu de culte marque véritablement le paysage local en raison de sa tour imposante (gōpuram) caractéristique des grands temples sud-indiens et de la gigantesque statue rituelle de Hanumān (« unique au monde » selon le site Internet du temple), qui s'élève à onze mètres de haut et qui est large de plus de quatre mètres.

Fig. 5 : Arrivée de fidèles dans le nouveau temple de Hanumān à Panchavadi (2013)

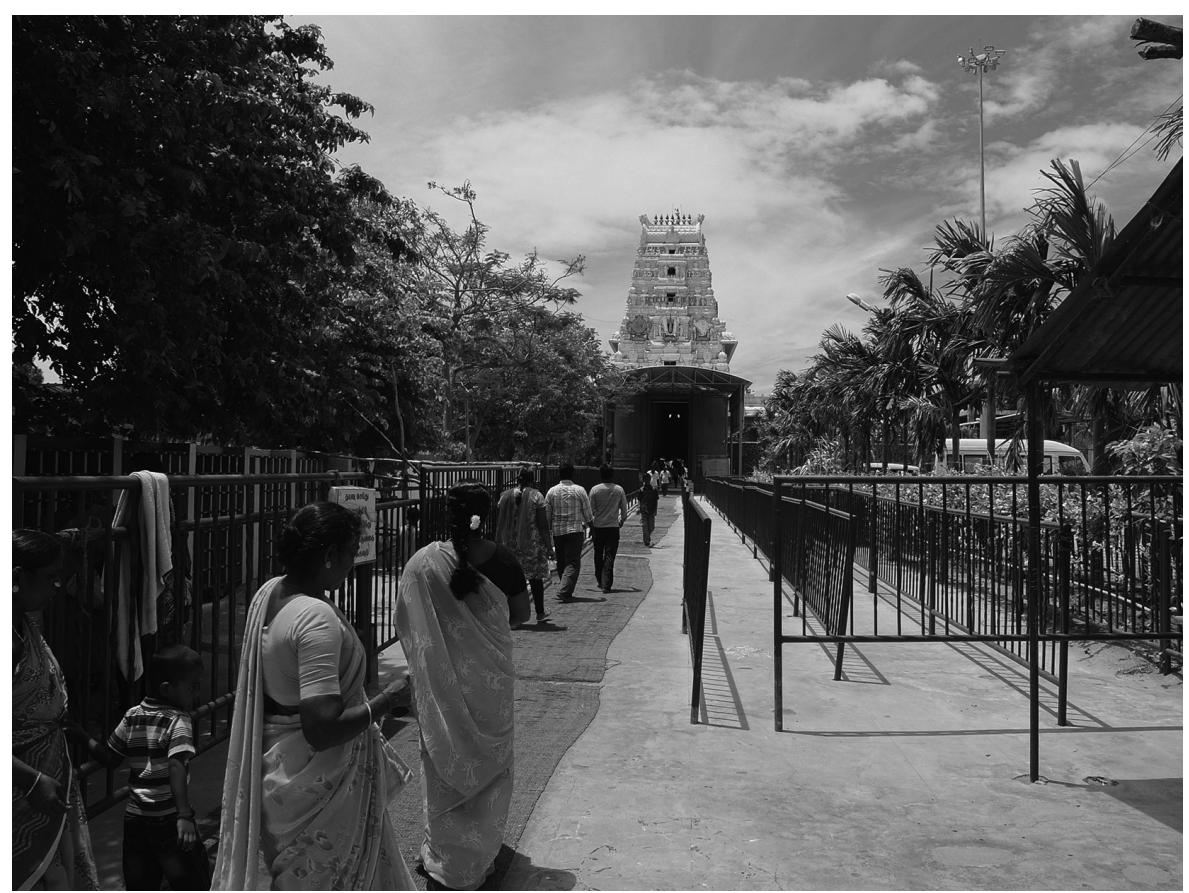

La réputation et le rayonnement de ce nouveau sanctuaire augmentent d'année en année, en résonance directe avec l'essor que connaît le culte de Hanumān depuis plusieurs décennies dans toute l'Inde ${ }^{30}$, où l'on assiste à

30. Dans la culture populaire pan-hindoue (et notamment grâce aux séries télévisées et autres bandes dessinées diffusées depuis les années 1980), Hanumān est reconnu comme le fidèle serviteur du dieuhéros Rām, dont l'image de roi divin est régulièrement instrumentalisée par les nationalistes hindous actuellement au pouvoir. Le dieu-singe incarne ainsi une certaine forme de patriotisme repris par les nationalistes, et qui transcende les différences de castes et de classes en raison de son culte moins orthodoxe - et donc plus universel - que ceux de Śiva ou Viṣnu. 
une véritable surenchère des fondateurs de temples qui cherchent régulièrement à construire la plus grande statue de Hanumān (Lutgendorf 1994 et 1997 ; Claveyrolas 2003). Et si les grands temples anciens dédiés au dieusinge sont assez rares en pays tamoul, son culte y est, comme ailleurs, en plein développement (Punzo-Waghorne 2004).

Le temple de Hanumān de Panchavadi doit son existence à deux individus. Tout d'abord (Sri) Ramani Anna, également connu au Tamil Nadu pour être le fondateur et gestionnaire (managing trustee) d'un autre grand temple de Hanumān situé à Chennai (Nanganallur). Ensuite, l'autre acteur majeur est Veeravalli S. Santhanam, un philanthrope cofondateur d'une importante compagnie de transport et de logistique basée à Chennai. Le premier a donc apporté la caution religieuse pour la construction du temple, alors que le second a assuré l'une des anciennes fonctions royales tamoules consistant à fournir des terres aux temples sous forme de donations. Ce dernier offrit en effet les cinq hectares du sanctuaire par le biais de sa société qui avait acheté un terrain à Panchavadi en 1999 pour y installer des entrepôts à proximité de l'échangeur autoroutier. La présence du temple dans ce village a ainsi été déterminée par celles de l'échangeur puis de la société du futur donateur du terrain. La solide réputation des deux hommes leur a également permis d'obtenir des fonds supplémentaires pour financer la construction du temple, estimée au final à 20 millions de roupies (275000 euros) ${ }^{31}$. L'inauguration rituelle (mahā-kumpapișēkam) du temple coûta à elle seule 600000 roupies (8 200 euros). Par ailleurs, le temple était administré en 2013 par douze ${ }^{32}$ gestionnaires (trustees) étant pour la plupart des notables de Chennai et de Pondichéry (magistrats, hauts fonctionnaires, chefs de grandes entreprises), ce qui confirme l'importance de l'évergétisme dans la création de ce temple. On notera également, avec Clémentin-Ojha et Lachaier (ibid.), qu'être trustee de la fondation d'un grand temple présente aussi l'avantage d'établir et de maintenir des liens interpersonnels de confiance qui facilitent l'échange d'informations entre membres et la mise en commun de ressources diverses.

Un des phénomènes les plus frappants à Panchavadi depuis la construction du temple de Hanumān concerne le développement immobilier s'étendant sur les terres alentour, qui étaient jusque-là essentiellement agricoles. Le temple a en effet apporté une dimension religieuse nouvelle à la localité - et donc de la valeur ajoutée -, pouvant amener la classe moyenne à s'y installer. C'est ainsi que de nombreux terrains agricoles ont été convertis, depuis, en lotissements résidentiels. Le principal programme de développement

31. «Hanuman's special avatar as Annihilator », The Hindu, 7 mars 2003.

32. Ce nombre élevé de trustees s'explique par le fait que deux fondations sont associées au temple. Une première administre les affaires rituelles du temple, et une seconde ses activités caritatives. 
immobilier a été lancé en 2011 par une première société. En juillet 2012, 57 lots étaient disponibles à proximité immédiate du temple et plusieurs habitations d'un niveau de standing correspondant aux attentes de la classe moyenne étaient déjà construites. Cette nouvelle zone résidentielle a été nommée Jaya Ram Nagar ( «ville de Rām le victorieux ») en référence aux divinités du nouveau grand temple - Hanumān étant le serviteur dévoué du dieu héros Rām -, ce qui témoigne de l'importance symbolique du lieu de culte pour l'identité de la localité. Assez curieusement, les promoteurs immobiliers n'ont aucun lien avec les fondateurs du temple, ni avec les trustees. Ils ont simplement repéré le potentiel de développement offert par cette localité située proche d'un échangeur et de la ville de Pondichéry, et surtout où un grand temple d'une divinité très populaire venait d'être fondé. D'ailleurs, les annonces publicitaires du lotissement ont pour principal argument la proximité immédiate du nouveau lieu de culte, ce qui est très siginificatif de la valeur ajoutée qu'il a apportée à la localité. Un deuxième programme immobilier de dimension comparable a été mené par une autre société dans le village adjacent de Sedarapet, ce qui confirme l'intérêt des promoteurs pour les environs du temple, et plus largement la relation étroite entre ce nouveau lieu de culte et le développement de sa localité. En 2013, il y avait également une douzaine de nouveaux magasins établis près de l'entrée du temple et plusieurs vendeuses d'offrandes rituelles destinées aux visiteurs du temple.

Pour autant, si la présence du temple a entraîné un développement immobilier remarquable à Panchavadi, la présence de la société de transport du philanthrope Santhanam n'a quant à elle pas eu d'impact sur l'emploi local, puisqu'elle n'y possède que des entrepôts. La plupart des habitants restent largement employés dans l'agriculture locale et les petites industries du village voisin de Sedarapet.

Ce temple de Hanumān a par ailleurs été construit à un endroit très surprenant. En effet, contre toute attente, il est situé dans un cêri, c'est-à-dire dans un hameau réservé aux ex-Intouchables en pays tamoul. La localisation du nouveau temple est donc complètement contraire au modèle classique de répartition spatiale de l'habitat des castes et des lieux des dieux, puisque les temples orthodoxes ayant des desservants brahmanes ne sont jamais situés dans ces quartiers en raison de "l'idéologie hindoue du pur et de l'impur » (Dumont 1966) qui définit les cêri comme des espaces «polluants», où les grands dieux de l'hindouisme ne sauraient s'installer ni les Brahmanes officier. Ce décalage se retrouve dans le fait que le seul autre temple de Panchavadi est un petit temple de déesse tutélaire (Māriyammañ) fréquenté 
seulement par des ex-Intouchables de caste paraiyar ${ }^{33}$. De même, le temple principal de la commune (panchayat village ${ }^{34}$ ) où est situé le village de Panchavadi n'est pratiqué que par les locaux (de toutes castes cette fois-ci). Il n'y a en outre aucune interaction rituelle, lors de fêtes religieuses ou de processions, entre le temple de Hanumān et les temples locaux plus anciens, ce qui est très révélateur du décalage entre la présence de ce nouveau grand temple orthodoxe et la société locale, dont il a pourtant transformé le paysage religieux.

Philip Lutgendorf a montré que la popularité croissante du culte de Hanumān depuis les années 1990 est étroitement liée au fort «développement de la classe moyenne indienne » (Lutgendorf, 1997: 325), particulièrement versée dans la dévotion envers le dieu-singe. Les processus en cours à Panchavadi s'inscrivent dans cette tendance, puisque le temple de Hanumān attire essentiellement des fidèles appartenant à la classe moyenne des villes environnantes, et très peu les habitants du village. En effet, et bien que les exIntouchables paraiyars soient autorisés à entrer dans le temple, la plupart des riverains interrogés dans la commune préfèrent se rendre aux temples plus anciens de la région, dont les vertus sont reconnues depuis plus longtemps et dont les donations n'iront pas, selon eux, « dans les poches des riches qui gèrent le temple de Hanumān ».

En résumé, ce nouveau temple a réellement entraîné un développement économique local, comme le suggèrent l'apparition de magasins autour du lieu de culte et surtout les programmes immobiliers. Mais la présence de ce nouveau temple a pour autre conséquence de reconfigurer la géographie sociale et rituelle locale, qui ne correspond plus aux configurations habituelles des localités tamoules.

\section{Un nouveau temple pour acheter des terres et créer de la centralité}

À quelques kilomètres de Panchavadi, le nouveau temple du village de Morattandi (850 habitants selon le recensement de 2011) confirme que les nouveaux temples hindous peuvent créer de la centralité, tout en montrant qu'ils peuvent aussi permettre à leur fondateur d'acheter des terres.

La Fondation Śri Lalithambigai Vēda Śivāgama fut créée en 2002 par Chidambaram Gurrukkal, un brahmane travaillant jusqu'alors dans un temple

\footnotetext{
33. La plus nombreuse caste d'ex-Intouchables du pays tamoul, dont le nom est à l'origine du mot «paria ».

34. Il s'agit du panchayat village de Rawthankuppam.
} 
de déesse de Pondichéry, et qui souhaitait fonder un temple dédié aux Neuf Planètes (navagrahā, tam. navakkirakam), plus particulièrement au dieu Śani (Saturne). Si la plupart des temples shivaïtes tamouls possèdent un petit autel dédié aux Neuf Planètes (dont Śani), ceux spécifiquement dédiés à ces divinités sont très rares au Tamil Nadu, alors que l'astrologie est pourtant très importante dans la vie sociale (notamment pour fixer les dates des mariages) et qu'elle y est même de plus en plus populaire (Guenzi 2013 ; Avdeef 2014). Ce nouveau temple devait donc être particulièrement attractif en raison de la rareté de ses divinités tutélaires.

Pour ce faire, Chidambaram Gurrukkal créa donc une fondation de temple et désigna comme trustees ses deux fils, également prêtres et astrologues, ainsi que leurs épouses. Il acheta un hectare de terrain pour le nouveau sanctuaire à Morattandi, situé également à proximité de l'échangeur autoroutier reliant Pondichéry et Chennai. La zone était auparavant occupée par des plantations de casuarinas, jusqu'à ce qu'un promoteur immobilier convertisse 25 hectares en lots. L'inauguration rituelle de ce temple dédié à Śani, officiellement sous le nom de «Śri Viśvarūpa Mahā Śanīśvara Bhagavān », eut lieu quatre ans plus tard, en 2006.

On retrouve derrière la construction de ce nouveau temple une idéologie de l'unique et du spectaculaire comparable à celle observée au temple de Hanumān de Panchavadi. En effet, les trustees de ce nouveau temple revendiquent la création de la «world tallest 27 feet [...] statue of Śani », comme chacun peut le lire sur les reçus de donation imprimés par la fondation. Le sanctuaire possède également une imposante statue de Vināyaka haute de plus de 4 mètres et recouverte d'or, ainsi que des statues des neuf planètes hautes de plus de 2 mètres, alors qu'elles dépassent rarement 60 centimètres dans les temples habituels. En outre, le « sanctuaire» (kṣetram) a été nommé «Śri Navagrahā Parihāra Kṣêtram », affirmant ainsi que les neuf planètes et l'astrologie pratiquée dans ce temple peuvent « soigner» (parihāra) les gens et leurs problèmes. Il y a donc une volonté évidente de la part des fondateurs et gestionnaires d'attirer le maximum de visiteurs dans ce nouveau temple. Même les noms des divinités sont écrits en hindi dans le temple (sous les mentions tamoules et anglaises), ce qui est particulièrement inhabituel au Tamil Nadu bien connu pour son opposition à l'usage de cette langue nord-indienne.

Sept ans après la consécration du temple, le projet apparaît comme une réussite, car le sanctuaire a bénéficié de l'intérêt croissant pour l'astrologie en Inde, de la rareté des temples de Śani et de l'accessibilité du lieu. En effet, la situation bien choisie de ce nouveau temple à quelques kilomètres de Pondichéry et très proche de l'échangeur de l'autoroute venant de Chennai 
Fig. 6 : Les statues imposantes de Śani (en haut) et de Vināyaka (en bas) dans le nouveau sanctuaire de Morattandi (2013)
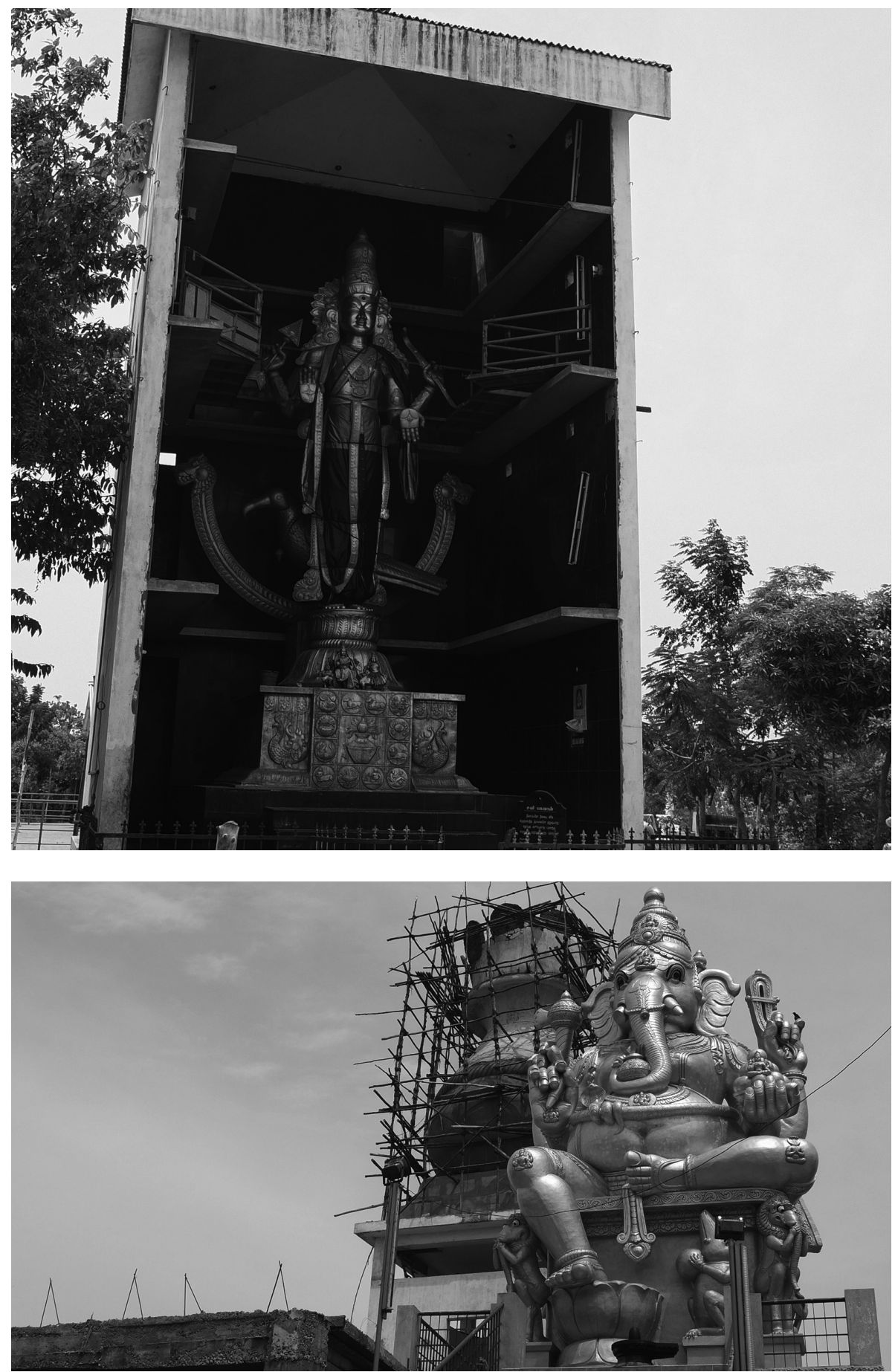
lui assure un réservoir de fidèles. Le péage de l'échangeur oblige en outre les conducteurs à s'arrêter à quelques centaines de mètres du temple, ce qui peut les motiver à s'y rendre. En 2013, grâce aux donations accumulées, la fondation du temple put même acheter sept autres parcelles à Moranttandi (dont une où elle a fondé une école de prêtres) et un hectare dans un village voisin.

Au final, tous les terrains achetés par la fondation du temple depuis 2002 représentent un bon investissement pour son fondateur puisque la valeur foncière de la zone a augmenté d'environ $50 \%$ en dix ans ${ }^{35}$, et qu'il semblait que cette tendance allait se poursuivre au vu des nombreuses maisons en cours de contruction en 2013 et du développement plus général des environs de l'échangeur. Si beaucoup de riverains dénoncent la stratégie spéculative du fondateur du temple qui a acheté des terres par l'intermédiaire de sa fondation religieuse, tous les propriétaires de Morattandi bénéficient néanmoins de l'augmentation de la valeur foncière. La présence du temple devrait par ailleurs pouvoir générer une nouvelle activité économique locale, selon une dynamique comparable à celle observée à Panchavadi. D'ailleurs, durant la grande fête religieuse dédiée à Śani (Śani peyarcci) en 2011, une informatrice locale put gagner 18000 roupies (250 euros) en une seule journée, grâce aux étals d'offrandes rituelles qu'elle avait installés pour l'occasion.

Le cas de Morattandi illustre ainsi un autre aspect majeur des potentialités économiques et de développement offertes par les fondations de temple en Inde : la législation des lieux de culte hindous permet à des investisseurs d'acheter de la terre par l'intermédiaire des fondations religieuses qui gèrent les temples. De plus, le nouveau temple crée une fois encore de la centralité par sa présence, ce qui contribue à l'émergence économique de sa localité.

\section{Développement local autour d'un temple de gourou}

Un dernier cas de figure concerne les temples et fondations récemment créés par des personnalités charismatiques telles que les maîtres spirituels ou gourous, reconnus comme des incarnations du divin. Cette configuration a été étudiée dans le bourg de Melmaruvathur, où le temple d'Ādi Parāsakti (déesse de l'énergie) et les fondations qui lui sont associées sont les principaux facteurs et acteurs du développement de la localité. Ce cas d'étude se distingue donc des exemples précédents par le profil de son fondateur, qui témoigne de l'immense popularité des gourous aujourd'hui en Inde et de l'importance des ressources économiques dont ils peuvent disposer, mais aussi par l'ampleur du développement local provoqué par la présence du

35. Selon des informateurs ayant participé au développement immobilier de la zone. 
nouveau temple. Il fournit également, et plus largement, un bon exemple de la création contemporaine de lieux de pèlerinage hindous.

En 2011, le bourg de Melmaruvathur était peuplé de 4459 habitants répartis sur deux communes (panchayat) adjacentes : celle de Melmaruvathur ellemême (1 748 habitants) et celle de Sothupakkam (2 711 habitants). Assez curieusement, ce gros village possède à lui seul deux gares routières, une gare ferroviaire, un hôpital, onze «high schools» et «colleges», et de nombreuses possibilités d'hébergements. Tout cela est dû essentiellement au temple d'Ādi Parāśakti, fondé en 1977 par le gourou Bangaru Adigalar (plus connu sous le nom d' $A m m \bar{a}^{36}$, ou « Mère » en tamoul, alors qu'il s'agit d'un homme), et qui connaît aujourd'hui une popularité dépassant largement les frontières de la localité.

Les rites pratiqués dans ce temple peuvent être associés à l'hindouisme dit « populaire ${ }^{37}$ » et au courant de la bhakti (« dévotion ») qui prône un rapport direct entre le fidèle et le divin. Le maître des lieux ne revendique d'ailleurs aucun lien avec les rites védiques, les prêtres ne sont pas brahmanes, et tous les fidèles sont autorisés à toucher la statue rituelle (mūlasthānam) du temple, ce qui n'est pas permis dans les temples orthodoxes. Le culte du temple et du gourou est ainsi particulièrement développé au sein des classes populaires (notamment rurales) du Tamil Nadu, sans pour autant leur être exclusif.

Le gourou lui-même n'est pas un brahmane (il est de caste naidu) et ne porte pas le cordon sacré ( $p \bar{u} n u l)$ des « hautes » castes. Il est né à Melmaruvathur en 1941 et aurait été, selon la légende, attiré par la méditation et la religion dès sa plus tendre enfance. En 1966, il y aurait découvert une pierre sacrée

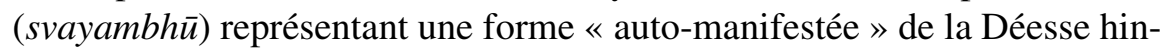
doue, symbolisant l'énergie divine. Comme l'indique le nom du temple, le gourou est reconnu depuis comme un avatār de Śakti (l' « énergie»), ce qui explique son surnom féminin.

Le cœur du temple ainsi que son pavillon principal (mandapam) ont été construits en 1977 à son initiative. L'année suivante, il créa la fondation Adhiparasakthi Charitable, Medical, Educational and Charitable Trust (ACMEC) puis, en 1988, l'Adhiparasakthi Siddhar Peetam Women's Charitable Trust (ASPWC). Ces fondations caritatives, dont il est le président et dont les objectifs portent essentiellement sur la santé et l'éducation, reçurent

\footnotetext{
36. Mais sans lien avec la «sainte» kéralaise Mata Amritanandamayi, plus célèbre en Occident et également surnommée «Ammā ».

37. C'est-à-dire ne respectant pas l'orthodoxie brahmanique de la « Grande » tradition hindoue (cf. note $11)$.
} 
d'importantes donations à mesure que la réputation du gourou et du lieu saint prit une ampleur régionale puis nationale dans les années 1980.

Grâce à ces dons abondants, les fondations ont créé de nombreux services, équipements et infrastructures de développement dans le village et au-delà. Les deux premières institutions mises en place par la fondation ACMEC ont été le Polytechnic College et le College of Pharmacy en 1983. Un Engineering College fut institué en 1984, puis une école et un collège l'année suivante. En 1986, la fondation ACMEC construisit le premier hôpital du village, qui peut accueillir aujourd'hui 1000 patients (et 1200 en ambulatoire). La gare ferroviaire fut également inaugurée en 1986 et le commissariat du village deux ans plus tard. Un College of Physiotherapy ouvrit en 1994, un Dental College et un foyer pour enfants handicapés en 2005, un lycée et un Institute of Medical Sciences and Research en 2007, puis une autre école en 2009.

Toutes ces institutions ont été fondées dans le village grâce à Bangaru Adigalar et son temple. Les institutions médicales et d'enseignement ont été directement créées par les fondations qu'il dirige, alors que les infrastructures de transport et le commissariat l'ont été par l'État du Tamil Nadu, qui a pris conscience du potentiel de développement de la localité dès les années 1980, mais aussi en raison des liens que le gourou a y su tisser ${ }^{38}$. L'ensemble atteste de la forte capacité d'attraction et de polarisation de ce temple récent.

En 2011, si la population officielle du bourg était de 4459 habitants d'après le recensement national, la population réellement présente au quotidien à Melmaruvathur grâce au temple est bien plus importante. En effet, les 3000 étudiants et les 55 médecins de l'hôpital (ainsi que leurs familles) qui résident à Melmaruvathur doivent notamment être considérés. Il en va de même pour les 50 à 250 bénévoles présents chaque jour pour aider au fonctionnement des activités religieuses et caritatives des fondations du temple. Par ailleurs, entre 2000 et 75000 repas sont offerts quotidiennement par le temple, ce qui constitue un bon indicateur du nombre de fidèles qui se rendent chaque jour à Melmaruvathur - le plus grand nombre de repas étant bien sûr offerts lors des fêtes religieuses. Enfin, les 1000 à 2200 patients de l'hôpital doivent aussi être pris en considération dans l'estimation de la population régulièrement présente dans la localité.

\footnotetext{
38. En particulier au sein du parti régional au pouvoir All India Anna Dravida Munnetra Kazhagam (AIADMK), et ce à tel point que Bangaru Adigalar fut surnommé dans la presse locale comme «le saint patron des politiciens de l'AIADMK » (ma traduction, Sunil, K. P. 1986, « The Godman Cometh », Illustrated Weekly of India (26 octobre), p. 52-5, cité par Fuller 2003 : 118)
} 
Fig. 7 : Pèlerins à l'entrée du temple du gourou (en haut) et l'Institute of Medical Sciences and Research créé par les fondations (en bas) (2013)
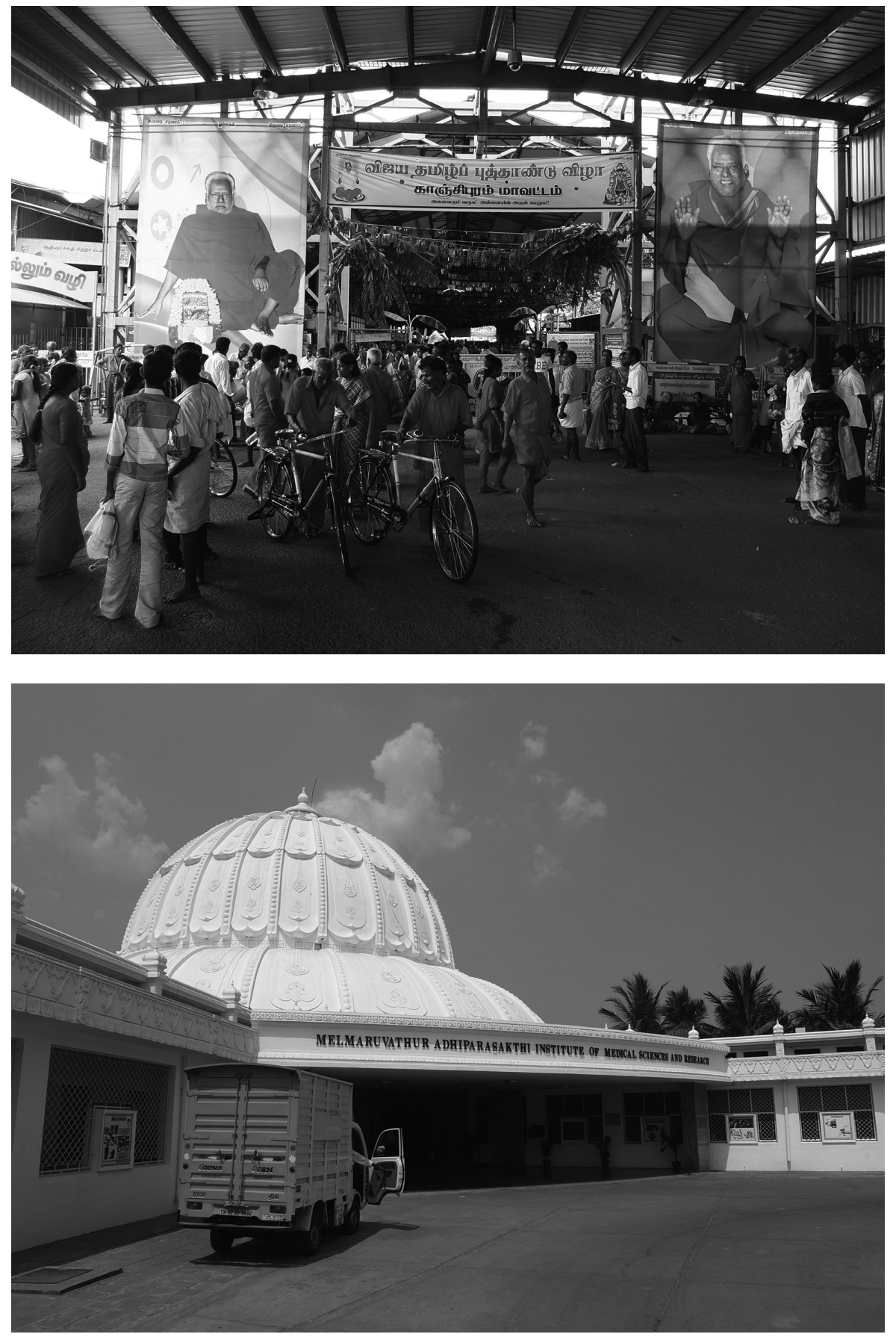
Comme beaucoup d'autres fondations de temple, celle du gourou de Melmaruvathur possède aussi des terres situées hors de la localité, ce qui lui permet de participer au développement d'autres territoires. La fondation ACMEC possède notamment des terres dans les environs de la petite ville de Kalavai (district de Vellore), située à environ 80 kilomètres de Melmaruvathur, où les 30 premiers hectares ont été donnés par le très influent Sankaracharya ${ }^{39}$ de Kanchipuram qui y possède un monastère. Comme à Melmaruvathur, elle y a également fondé plusieurs universités, à commencer par le College of Arts \& Science dont la première pierre fut posée en 1988 par l'ancien Premier ministre Rajiv Gandhi, ce qui confirme la réputation notable du gourou auprès des politiques ${ }^{40}$. La fondation y a aussi ouvert le premier Agricultural College auto-financé du Tamil Nadu en 1999, ainsi qu'un Teacher Training Institute en 2004 et un College of Education en 2005. La fondation ASPWC a quant à elle créé un College of Engineering en 2001.

Le bourg de Melmaruvathur est également connecté à la diaspora et à la mondialisation grâce à son temple. En effet, au-delà du Tamil Nadu, le temple et les fondations qui lui sont associées reçoivent des visiteurs et des dons de toutes parts de l'Inde, mais aussi des États-Unis, du Canada, du RoyaumeUni, de France, de Singapour, d'Australie et de Dubai. La fondation se targue également de gérer 5000 associations religieuses (appelées « mandram ») présentes à différents endroits du globe (dont Paris) et où le gourou se rend plus ou moins régulièrement.

Au final, bien que l'accessibilité du bourg depuis Chennai et d'autres centres urbains ne doive pas être sous-estimée dans le processus de développement local, ce temple et ses fondations apparaissent clairement comme étant à l'origine de l'émergence d'une centralité. Le cas de Melmaruvathur illustre donc parfaitement comment certains temples hindous et leurs fondations peuvent provoquer un développement local, avec en l'occurrence la création d'un grand centre religieux, médical et d'enseignement supérieur.

Ce phénomène n'est pas une exception puisqu'on le retrouve à d'autres endroits en Inde. C'est par exemple le cas dans le village de Kokamthan (district d'Ahmednagar, dans le Maharashtra), où la fondation du gourou Vishwatmak Jangli Maharaj a créé dix écoles, un hôpital, un Doctorate of Education College, un Center for Wrestling Education et une école de musique. Ces activités ne sont pas davantage réservées aux fondations récentes de gourous. Par exemple, le grand temple de Palani, qui date du $\mathrm{IX}^{\mathrm{e}}$ siècle et qui

\section{Abbé.}

40. Le parti du Congrès national indien (Indian National Congress), dont faisait partie Rajiv Gandhi, est bien entendu distinct du parti régional tamoul AIADMK évoqué dans la note 38. 
est totalement géré par l'État du Tamil $\mathrm{Nadu}^{41}$, administre quatre colleges, trois écoles, deux écoles de prêtres, un hôpital et un foyer pour enfants. Enfin, ce type de développement local se retrouve aussi autour de gourous décédés, comme l'ont notamment montré Shinde et Pinkney (2013) dans leur étude du village de Shirdi (Maharashtra) où vécut le saint Sai Baba (1832-1918), et qui est devenu en moins d'un siècle un centre de pèlerinage de rayonnement pan-indien, voire mondial.

\section{- Conclusion}

Alors que les temples hindous sont unanimement considérés par les historiens comme des acteurs majeurs de l'expansion urbaine dans l'Inde du Sud médiévale, le rôle de ces institutions religieuses dans le développement des localités a été négligé par les études urbaines récentes. Pourtant, certains temples peuvent être considérés comme de véritables acteurs du développement local dans l'Inde contemporaine, notamment parce que toutes les divinités de temple sont dotées d'une personnalité juridique et qu'on leur offre des ressources économiques et des propriétés foncières gérées par des fondations religieuses.

Comme ce fut le cas pendant des siècles, les temples du sud de l'Inde participent au développement de leur localité de différentes manières. Ils leur donnent une identité symbolique spécifique, attirent des visiteurs et des investisseurs de l'extérieur, participent à la structuration de la morphologie urbaine, influencent l'usage du sol au travers d'interdits spatiaux, et possèdent du foncier sur lesquel des activités économiques sont souvent installées.

De plus, les cas d'études considérés ici montrent que les temples actuels sont capables de participer au développement et à la croissance des bourgs, et parfois même de les provoquer. C'est le cas à Melmaruvathur et Panchavadi, où l'émergence d'activités économiques (petits commerces, programmes immobiliers) et d'infrastructures de développement (écoles, colleges, hôpitaux) a été déclenchée par la construction d'un temple. Ces exemples rappellent ainsi l'importance de l' "interdit spatial de propriété » associé aux lieux sacrés dans l'émergence et la structuration des établissements humains, comme le propose la géographie structurale. En effet, les terres sur lesquelles sont fondés les temples hindous n'appartiennent légalement qu'à leur divinité et leur

41. Il s'agit même du temple public générant chaque année, et de loin, les plus importantes recettes de l'État du Tamil Nadu. 


\section{Les temples hindous et le développement local en Inde du Sud}

sont inaliénables, mais ces lieux sont capables de motiver le développement de leur localité et de structurer leur environnement immédiat.

Enfin, le rôle des temples et de leurs fondations dans les stratégies de développement des investisseurs mérite également attention. L'exemple de Panchavadi montre en effet qu'un temple peut entraîner un développement immobilier dans ses environs, et celui de Morattandi qu'un fondateur de temple peut acheter des terres exonérées d'impôt pour sa fondation religieuse. Ces stratégies que l'on a souhaité étudier ici dans des bourgs et des petites villes, sont également observables dans des agglomérations bien plus importantes du sud de l'Inde. C'est notamment le cas à Bangalore, où une fondation caritative de la célèbre International Society for Krishna Consciousness (ISKCON, connue aussi sous le nom du mouvement Hare Krishna) développe actuellement tout un quartier (township) qui incluera des centaines de logements résidentiels ainsi qu'un centre commercial et un centre d'affaires, autour d'un grand complexe touristique et religieux dédié au dieu Krishna. Le but de l'opération est bien sûr de générer des revenus pour promouvoir les objectifs de la fondation ${ }^{42}$. Ceci confirme que si les études urbaines se sont peu intéressées à l'influence des temples sur le développement local, beaucoup d'acteurs et d'investisseurs sont en revanche pleinement conscients des possibilités offertes par les temples hindous et les fondations religieuses pour faire des affaires et développer les territoires en Inde du sud.

\section{$\nabla$ Bibliographie}

Annoussamy (2001), Le droit indien en marche, Paris : Société de législation comparée.

Appadurai Arjun ; Breckenridge, Carol (1976), « The South Indian Temple : Authority, Honour and Redistribution ». Contributions to Indian Sociology, 10, p. 187-211.

Avdeeff, Alexis (2014), Les feuilles de palme et le stylet. L'art de la prédiction astrologique chez les Valluvar du pays tamoul (Inde du Sud), thèse doctorat non publiée, Toulouse : EHESS.

Business Standard, « Temple trusts chant new investment mantras », Namrata Acharya, Feb 06, 2010.

Claveyrolas, Mathieu (2003), Quand le temple prend vie. Atmosphère et dévotion à Bénarès, Paris : Éditions du CNRS, coll. « Monde Indien ».

Clémentin-0jha, Catherine (2010), "L'insertion des convictions religieuses dans les droits positifs contemporains: le cas de l'Union indienne », présenté lors de la conférence Convictions philosophiques et religieuses et droits positifs, Centre international de la Common Law en français, Moncton, (24-27/08/2008), p. 324-366.

42. « Temple trusts chant new investment mantras », Namrata Acharya, Business Standard, 6 Fevrier 2010 . 
Clémentin-0jha Catherine \& Pierre Lachaier (dir.) (2008), Divines Richesses. Religion et économie en monde marchand indien, Paris : EFEO.

Colas, Gérard (2009), "Images and Territory of Gods : From Precepts to Epigraphs », in Berti Daniela et Gilles Tarabout (dir.) Territory, Soil and Society in South Asia, New Delhi : Manohar, p. 99-139.

Das Tuhin K. \& Ishitta Data Ray (2008), « Monopolistic Competitive Market in Religion : A Case Study of Hindu Temples », Applied Economic Letters, Vol. 15 Issue 1, p. 69-72.

Denis, Eric ; Mukhopadhyay, Partha ; Zérah, Marie-Hélène (2012), « Subaltern Urbanisation in India », Economic \& Political Weekly, July 28, vol xlvii, n³0, p. 52-62.

Desmarais, Gaëtan (2001), « Pour une géographie humaine structurale/Elements of structural geography », Annales de Géographie, t. 110, n 617, p. 3-21.

Dumont, Louis (1966), Homo hierarchicus. Essai sur le système des castes et ses implications. Paris : Gallimard.

Eck, Diana L. (2012), India. A Sacred Geography, New-York : Three Rivers Press.

Fuller, Christopher J. (2003), The Renewal of the Priesthood. Modernity and Traditionalism in a South Indian Temple. New Delhi : 0xford University Press.

Gaucher, Jacques (2007), De la maison à la ville en pays tamoul ou la diagonale interdite. Enquête sur les formes urbaines de la ville-temple sud-indienne. Paris : Éditions de l'École Française d'Extrême Orient.

Geddes, Patrick (1976) [1919], "The Temple City », in Ferreira J.S. \& S.S. Jha (eds.), The Outlook Tower: Essay on Urbanization in Memory of Patrick Geddes, Bombay : Popular Prakashan, p.461-475.

Guenzi, Caterina (2013), Le discours du destin. La pratique de l'astrologie à Bénarès. Paris : CNRS Éditions.

Guilmoto, Christophe ; Reiniche Marie-Louise ; Pichard, Pierre (1990), Tiruvanamalai : un lieu saint sivaïte du sud de l'Inde. Pondichéry : Éditions de l'École Française d'Extrême-Orient, volume 5 « la ville».

Heitzman, James (1987), « Temple urbanism in medieval South India », The Journal of Asian Studies, Vol. 46, $\mathrm{n}^{\circ} 4$ (Nov.), p. 791-826.

Iannacone, Laurence (1998), "Introduction to the Economics of Religion », Journal of Economic Literature, Vol. XXXVI, p. 1465-1496.

Louiset, Odette (2011), L'oubli des villes de l'Inde, Paris: Armand Colin-Recherches.

Lutgendorf, Philip (1994), « My Hanuman is bigger than yours ». History of Religions, 33-3, p. 211-245.

Lutgendorf, Philip (1997), « Monkey in the Middle : The Status of Hanuman in Popular Hinduism. » Religion, 27, p. 311-332.

Mukherjea, Bijan Kumar (2003) [1952], The Hindu law of Religious and Charitable Trust, Calcutta : Eastern Law House Ltd.

Narayanan, Yamini (2015), Religion, Heritage and the Sustainable City: Hinduism and Urbanisation in Jaipur, London, Routlegde.

Obadia, Lionel (2013), La marchandisation de Dieu. L'économie religieuse. Paris: CNRS Éditions. 


\section{Les temples hindous et le développement local en Inde du Sud}

Presler, Franklin A. (1983), The Structure and Consequences of Temple Policy in Tamil Nadu, 1967-81, Pacific Affairs, Vol. 56, No. 2 (Summer, 1983), p. 232-246.

Presler, Frankin A. (1987), Religion Under Bureaucracy : Policy and Administration for Hindu Temples in South India. Cambridge : Cambridge University Press.

Punzo-Waghorne, Joanne (2004), Diaspora of the Gods. Modern Hindu temples in an Urban Middle-Class World. New York : Oxford University Press.

Reiniche, Marie-Louise (1989), Tiruvanamalai : un lieu saint sivaïte du sud de l'Inde. Pondichéry : Éditions de l'École Française d'Extrême-Orient, volume 4 « La configuration sociologique du temple hindou ».

Reiniche, Marie-Louise (1985), « Le Temple dans la localité : Quatre exemples au Tamilnad. » in Jean-Claude Galey (dir), L'Espace du temple 1. Paris : Éditions de l'EHESS, Coll. Purusāartha, $\mathrm{n}^{\circ} 8, \mathrm{p} .75-121$.

Shinde Kiran A. \& Andrea M. Pinkney (2013), "Shirdi in Transition: Guru Devotion, Urbanisation and Regional Pluralism in India », South Asia: Journal of South Asian Studies, $36: 4$, p. 554-570.

Singer, Milton (1972), « Industrial Leadership, the Hindu Ethic, and the Spirit of Socialism », in When a Great Tradition Modernizes. An Anthropological Approach to Indian Civilization, Delhi : Vikas Publishing House, p. 272-382.

Stein, Burton (1961), « The State, the temple and agricultural development: A study in Medieval South India », Economic Weekly Annual, vol. XIII, n 4-5-6, Jan. 28, p. 179-187.

Stein, Burton (1960), « The Economic Function of a Medieval South Indian Temple », Journal of Asian Studies, vol. XIX, p. 163-176.

Tamil Development, Religious Endowments and Information Department, Hindu Religious and Charitable Endowments Department, 2014, Demand N 47, Policy note 2014-2015.

Tarabout, Gilles (2009), «The Annamanada Case : A Hundred Yeras of Conflict over Rights and Territories in Kerala », in Berti Daniela et Gilles Tarabout (dir.) Territory, Soil and Society in South Asia, New Delhi : Manohar, p. 271-310.

Tarabout, Gilles (2006) [1996], "Les transformations de l'hindouisme », in Christophe Jaffrelot (dir.) L'Inde contemporaine. De 1950 à nos jours, Paris : Fayard, CERI, p. 568-579.

The Hindu (2003), « Hanuman's special avatar as Annihilator », Friday, Mar 07.

Varadachari V. K. (1968), The law of Hindu Religious and Charitable Endowments. Lucknow : Eastern book Co.

Veyne, Paul (1976), Le Pain et le Cirque : Sociologie historique d'un pluralisme politique. Paris : Éditions du Seuil.

Viguier, Anne (2007), « Images et formes urbaines : L'héritage précolonial du pays tamoul. », in Dupont Véronique et Djallal G. Heuzé (dir.) La ville en Asie du Sud: Analyse et mise en perspective, Paris : Éditions de l'EHESS, Collection/revue « Purusartha », $\mathrm{n}^{\circ} 26, \mathrm{p} .43-77$. 